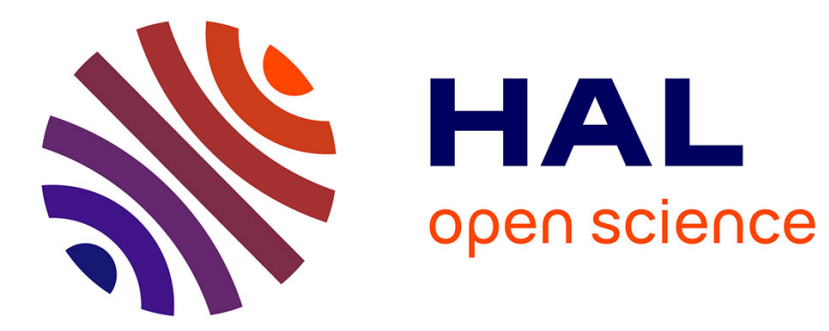

\title{
Graph analysis of uterine networks using EHG source connectivity
}

Noujoud Nader, Saeed Zahran, Catherine Marque, Mahmoud Hassan, Maxime Yochum, Wassim Falou, Mohamad Khalil

\section{- To cite this version:}

Noujoud Nader, Saeed Zahran, Catherine Marque, Mahmoud Hassan, Maxime Yochum, et al.. Graph analysis of uterine networks using EHG source connectivity. 2017. hal-01544687

\section{HAL Id: hal-01544687 \\ https://hal.science/hal-01544687}

Preprint submitted on 22 Jun 2017

HAL is a multi-disciplinary open access archive for the deposit and dissemination of scientific research documents, whether they are published or not. The documents may come from teaching and research institutions in France or abroad, or from public or private research centers.
L'archive ouverte pluridisciplinaire HAL, est destinée au dépôt et à la diffusion de documents scientifiques de niveau recherche, publiés ou non, émanant des établissements d'enseignement et de recherche français ou étrangers, des laboratoires publics ou privés. 


\title{
Graph analysis of uterine networks using EHG source connectivity
}

\author{
Noujoud Nader, Saeed Zahran, Catherine Marque \\ CNRS UMR 7338, BMBI \\ Sorbonne University, Université de technologie de \\ Compiègne, Compiègne, France \\ noujoud.nader@utc.fr, Catherine.marque@utc.fr, \\ saeed.zahran@utc.fr
}

\author{
Mahmoud Hassan, Maxime Yochum \\ Laboratoire Traitement du Signal et de L'image, INSERM \\ Université de Rennes 1 \\ Rennes, France \\ mahmoud.hassan@univ-rennes1.fr, maxime.yochum@univ- \\ rennes1.fr
}

\author{
Noujoud Nader, Wassim Falou, Mohamad Khalil \\ Azm Center in Biotechnology and its application, Lebanese University \\ Tripoli, Lebanon \\ wafalou@ul.edu.lb, Mohamad.khalil@ul.edu.lb
}

\begin{abstract}
Emerging evidence show that the connectivity analysis of the uterine signals is a powerful tool in characterizing pregnancy and labor contractions. Here, we present the results of studying the connectivity between uterine sources identified from the electrohysterogram (EHG) signals, which reflects the electrical activity of the uterine muscle. We started by evaluating the effect of the two key steps involved in EHG source connectivity processing: i) the algorithm used in the solution of the inverse problem and ii) the method used for the estimation of the functional connectivity. We evaluate three different inverse solutions (to reconstruct the dynamic of uterine sources) and three connectivity measures (to compute statistical coupling between the reconstructed sources). The networks obtained by each combination of the inverse/connectivity methods were compared to a reference network (ground truth) generated by the model. The method was then applied to real EHG signals in order to discriminate pregnancy and labor contractions.
\end{abstract}

Keywords-Uterine sources; electrohysterogram EHG; forward/inverse problem; network analysis I. INTRODUCTION

"Every object that biology studies is a system of systems" [1]. Among the complex system of systems of the human body, many questions remain open concerning the human uterus. One of the most promising markers of the uterine contractions is the electrical activity of the uterus. This activity is reflected in the electrohysterogram (EHG), which is a noninvasive abdominal measurement of the uterine electrical activity [2]. Delivery is preceded by two physiological phenomena: increased excitability and increased connectivity among the myometrial cells resulting in increased propagation of the action potentials that underlie uterine contractions [2]. Thus, numerous studies, based on the analysis of the propagation (or synchronization) of the uterine signals, have shown its power in characterizing uterine activity and discriminating pregnancy and labor contractions [3].

Several studies have been realized to characterize the uterine propagation by means of the synchronization between EHG signals recorded at the abdominal surface. These efforts were based on various methods such as i) correlation/connectivity analysis [4], [5] where the methods were applied on the entire uterine burst manually segmented, and ii) propagation velocity quantified by analyzing either the propagation of whole bursts of EHG [6], or of single spikes identified within bursts [7].

The original feature of the present work is to study the uterine connectivity at the uterine electrical sources. Briefly, the method consists of reconstructing the dynamic of uterine sources by solving the inverse problem and then compute the connectivity between these reconstructed time series. First, we test the methods on simulated data (ground truth) generated by using a biophysically plausible model developed in our team. By changing the inverse and connectivity methods, we compared with the reference network the networks obtained by each of the combination. Uterine networks then were estimated for contractions recorded during pregnancy and labor. A network-based analysis was performed to compute the significant differences between both conditions. This analysis was done at a node-wise level (computing the difference at each uterine source).

\section{MATERIALS AND METHODS}

\section{A. Inverse problem}

The uterine sources have first to be estimated from the surface-level recordings, the EHGs, by solving the so-called inverse problem. Generally speaking, this inverse problem consists of estimating the internal sources $\mathrm{S}(\mathrm{t})$ from the surface signals $\mathrm{X}(\mathrm{t})$ (here the EHGs). The main advantage of this approach is to analyze directly the sources that generate the EHG signals. Source reconstruction has been widely applied to EEG [8] and MEG [9]. To our knowledge, the source localization has been applied on uterine EHG very recently, for the first time, by Marque et al. [10]. Source localization requires two processing steps: i) the forward problem, to model the path from the source to the surface signals; ii) the inverse problem, going from real surface signals to the estimated sources.

The forward problem was solved by using the boundary element method (BEM) with OpenMEEG [11] 


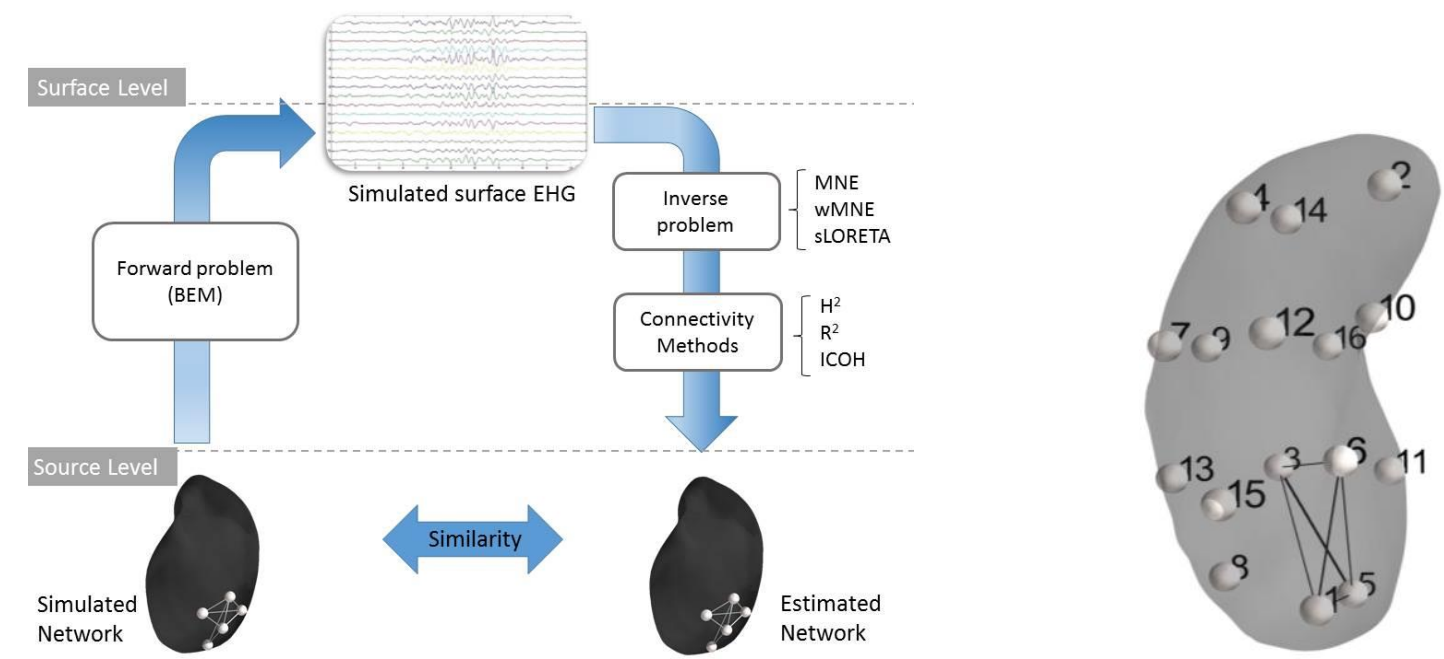

Figure 1: A) Structure of the investigation. First, a given network is generated by the model and considered as the 'ground truth'. The statistical couplings are then computed between the original sources by using three connectivity methods $\left(R^{2}, h^{2}\right.$ and Icoh). By solving the forward problem, we generate synthetic EHGs. These signals are then used to solve the inverse problem by using three inverse solutions (MNE, wMNE, sLORETA). The statistical couplings are then computed between the reconstructed sources by using the same connectivity methods $\left(R^{2}, h^{2}\right.$ and Icoh). The identified network by each combination (inverse/connectivity) was then compared with the original network using a 'network similarity' algorithm. B) Simulated Network

According to the linear discrete equivalent of current dipole model, EHG signals $\mathrm{X}(\mathrm{t})$ measured from $\mathrm{M}$ channels can be expressed as linear combinations of $\mathrm{P}$ time-varying current dipole sources $S(t)$ as follow:

$X(t)=G \cdot S(t)+N(t)$

where $G$ is the leadfield matrix of the dipolar sources and $N(t)$ an additive noise. The inverse problem consists of finding an estimate $\widehat{S}(t)$ of the dipolar source parameters given $X(t)$ and $\boldsymbol{G}$, already computed using BEM by means of the forward problem step. We used here three of the most classically used algorithms to solve the inverse problem: Minimum Norm Estimate $(M N E)$, weighted Minimum Norm Estimate ( $w M N E)$ and the standardized Low Resolution brain Electromagnetic Tomography (sLORETA) [8].

\section{B. Data}

Real Data: The signals were recorded from 16 monopolar channels of a $4 \times 4$ matrix located on the woman's abdomen. The third electrode column was always put on the uterine median vertical axis (see [12] for more details). The sampling frequency was $200 \mathrm{~Hz}$. The data were recorded at the Landspitali university hospital (Iceland) using a protocol agreed by the relevant ethical committee (VSN02-0006-V2) and at the Center for Obstetrics and Gynecology (Amiens, France), using a protocol agreed by the relevant ethical committee (ID-RCB 2011-A00500-41). The EHG signals were manually segmented and denoised by using a CCA-EMD method developed in our team [13]. After segmentation and denoising, we obtained 183 labor and 247 pregnancy EHG bursts, associated to contractions, at different weeks of gestation. The analysis below has been applied to these segmented uterine bursts.

Simulated Data: We have used a realistic model developed in our team, to simulate EHGs [14]. This model permits us to control the number, position and activity of the uterine sources (network of sources) used to simulate the EHGs. The original simulated network is called the "ground truth". The simulation time was 45 seconds and the sampling frequency was $200 \mathrm{~Hz}$. In our case we have activated 1000 cells that were grouped into sixteen zones depending on their Euclidian distance. We have labeled these zones by their number (from 1 to 16). Only four zones were activated (zones 1, 3, 5 and 6). The signals of zones 1 and 6 started at $\mathrm{t} 0=0 \mathrm{~s}$, while a time delay was added to signals of zones 3 and 5 . Zones 3 and 5 were highly correlated with zones 1 and 6, but with a delay (30s). (Figure 1B).

C. OVERVIEW

As illustrated in Figure 1A, a given simulated network was generated at the source level. Surface EHG signals were obtained by solving the forward problem. The volume conductor contains: the myometrium (where the source is located) with conductivity $=0.2 \mathrm{~S} / \mathrm{m}$ and depth $=0$ (all the sources are located at the uterine surface); the abdominal muscle with conductivity $0.3 \mathrm{~S} / \mathrm{m}$, and thickness $=0.936 \mathrm{~cm}$; fat with conductivity $=0.04 \mathrm{~S} / \mathrm{m}$ and thickness $=2 \mathrm{~cm}$; and skin with conductivity $=0.5 \mathrm{~S} / \mathrm{m}$ and thickness $=0.2 \mathrm{~cm}$. The corresponding leadfield is computed by using the BEM with OpenMEEG [11]. After the estimation of EHG signals we added to these signals different SNR values. Sources were then estimated by using inverse solution algorithms (wMNE, sLORETA and MNE). After that, functional connectivity was estimated by using three methods $\left(R^{2}, h^{2}, I c o h\right)$. The connectivity matrices were $16 \times 16$. These matrices were thresholded by saving edges with the highest weight values. In order to compare the reference uterine network with the network identified by each of the inverse/connectivity combination, we used the simNet algorithm [15]. This algorithm provides a normalized Similarity Index (SI) between 0 (totally different graph) and 1 (same graph).

\section{MEASURING THE FUNCTIONAL CONNECTIVITY}

We have quantified the correlation using three well know connectivity methods: the linear correlation coefficient $\left(R^{2}\right)$ the nonlinear correlation coefficient $\left(h^{2}\right)$ and the imaginary part of coherence (Icoh). 


\section{E. GRAPH METRICS}

To quantify the graph connectivity, we have used in this work one parameter classically used to characterize graph connectivity, the Strength (Str). This parameter quantifies the importance and contribution of each node with respect to the rest of the network.

$$
\mathrm{S}_{\mathrm{i}}=\sum_{\mathrm{j} \in \mathbb{N}} \mathrm{w}_{\mathrm{ij}} \text { - }
$$

where $\mathrm{i}, \mathrm{j}$ denotes respectively the $\mathrm{i}^{\text {th }}, \mathrm{j}^{\text {th }}$ nodes, and $\mathrm{w}_{\mathrm{ij}}$ is the value (Icoh value) of the relation between nodes $i$ and $j$ [16].

\section{RESULTS}

\section{A. Results on simulated data}

The results obtained on the simulated data are illustrated Figure 2. A visual analysis shows that the number of connections between the different zones vary according to the combination of methods used. For a given connectivity approach, changing the localization method modify more or less the network, depending on the connectivity method. On the other hand, for a given source localization approach, the functional connectivity measure changes qualitatively the network only for Icoh. $h^{2}$ or $R^{2}$ combined with sLORETA give the network that best matches the reference network. Overall, values of the Similarity Index are low (16\% to 27\%). Results obtained by using $h^{2}$ are on average better than by using $R^{2}$ and Icoh. The combination providing the highest similarity is sLORETA $/ h^{2}(27.8 \%)$, followed by sLORETA/R ${ }^{2}(27.7 \%)$ and $\mathrm{wMNE} / \mathrm{h}^{2}(27 \%)$. Icoh gives the lowest similarity whatever the localization algorithm. The results obtained with sLORETA $/ \mathrm{h}^{2}$ and sLORETA $/ \mathrm{R}^{2}$ are significantly closer to the reference network than the other ones (Wilcoxon rank-sum test, $\mathrm{p}<0.01$, corrected using Bonferroni).

\section{B. Results on real data}

We then applied the EHG source connectivity methods to real EHG data. The main motivation is to find a possible significant difference (at node level) between networks obtained for pregnancy and labor contractions. As no one combination of inverse/connectivity methods arose from the simulation-based analysis (described above), we applied all the combinations on real EHG (segmented and denoised bursts). In this analysis we compute the Str for each zone. We then performed a statistical test at the level of each node (each zone) between pregnancy and labor networks. We plot for each inverse/connectivity combination method only the zones that present a difference between labor and pregnancy. Figure 3 shows the different zones that present a significant difference between labor and pregnancy when using Str as graph measure. All the nodes presented in this figure have $\mathrm{p}$ value $<0.01$ using Wilcoxon test, corrected for multiple comparison using Bonferroni method. Results show that, when using $\mathrm{h}^{2}$ as a connectivity method, the number of significant zones $(6 / 16)$ is the same whatever the inverse problem method used. The lowest p_value is obtained when using the wMNE for zone $8\left(\mathrm{p}=1.2710^{-30}\right) . \mathrm{R}^{2}$ gave the highest number of zones combined with sLORETA (10 zones) and with wMNE (9 zones). The lowest $\mathrm{p}$ _value is given for zone 8 with wMNE $\left(\mathrm{p}=6.6910^{-27}\right)$. Only one zone (zone 16$)$ provided a significant difference when using $\mathrm{MNE} / \mathrm{Icoh}\left(\mathrm{p}=4.410^{-4}\right)$, while no efficient zones are given with wMNE/Icoh and sLORETA/Icoh.

\section{DISCUSSION AND CONCLUSION}

In this paper, we presented the preliminary results of a novel approach aiming at characterizing the EHG functional connectivity at the source level. The originality in this work is the use of a network-based uterine source level analysis to study the synchronization between uterine electrical activity, in clinical perspective. We reported a comparative study of the networks obtained from all possible combinations between

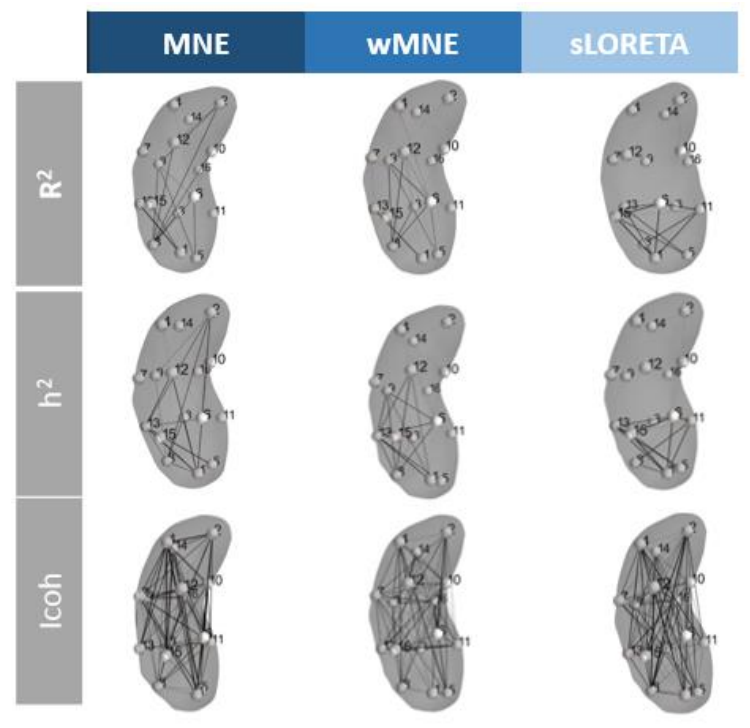

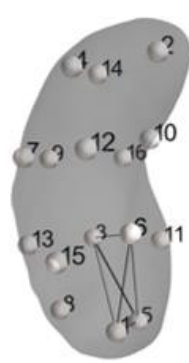

Ground truth

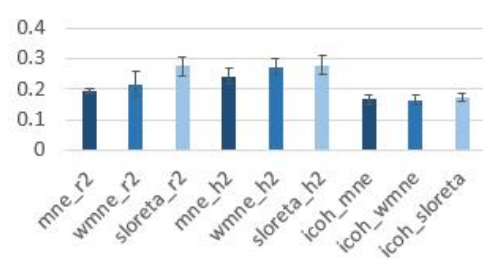

C Similarity Index

Figure.1. A) Uterine networks obtained by using the different inverse and connectivity methods, B) The original network (ground truth) and C) Values (mean \pm standard deviation) of the similarity indices computed between the network identified by each combination and the model network. 


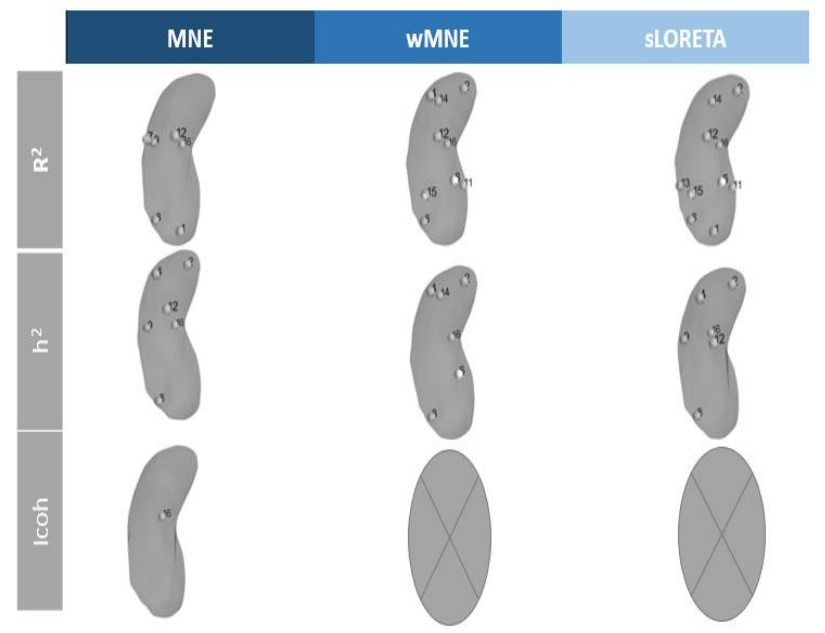

Figure 3. Node-wise analysis for Strength metric. Only nodes showing significant differences between pregnancy/labor were visualized

three algorithms to solve the EHG inverse problem and three methods to estimate the functional connectivity. A second originality of this study is related to the use of simulated EHG signals from a realistic uterine model, as a ground truth to compare the performance of the studied methods.

Results obtained on the simulated data indicated that more than one combination give relevant networks when compared with the ground-truth. Indeed, sLORETA combined with $\mathrm{h}^{2}$ or $\mathrm{R}^{2}$ gives best results but the similarity indexes are low. Thus, we have applied on real EHGs all the possible combinations. The obtained results indicate that $w M N E$ combined with $R^{2}$ or $h^{2}$ gives better results than the other combinations.

\section{Methodological consideration}

The connectivity matrices were thresholded by keeping the edges with the highest weight values (strongest 10\%). We were aware of a possible effect of this threshold. Several threshold values $(50 \%$ to the $5 \%$ ) were tested and results were relatively consistent across threshold.

Three classical inverse and connectivity algorithms were evaluated here. We focused this study on evaluating 'functional' connectivity methods. Nevertheless, the analyse of 'effective' connectivity methods that investigate the causality between different active zones, may be of interest in order to study the propagation direction in labor and pregnancy. In addition, using other inverse methods more suited to the uterine activity (currently under study) will be of great interest to improve these preliminary results.

The uterus model used in this study was computed by using the BEM method with four tissue layers. Nevertheless, other methods to solve the forward model such as the Finite Element Method (FEM) are under investigation.

\section{Node wise analysis}

The strength graph metric showed several significant zones that differentiate between pregnancy and labor. This parameter (and other graph measures) will be used as features for classification purpose (using machine learning approaches for instance) to test its performance in detecting labor or predicting premature labor when such data are available.

\section{REFERENCES}

[1] F. Jacob, The logic of life: a history of heredity. Vintage Books, 1976.

[2] D. Devedeux, C. Marque, S. Mansour, G. Germain, and J. Duchêne, "Uterine electromyography: a critical review," Am. J. Obstet. Gynecol., vol. 169, no. 6, pp. 1636-1653, Dec. 1993.

[3] M. M. Hassan, J. Terrien, C. Muszynski, A. Alexandersson, C. Marque, and B. Karlsson, "Better Pregnancy Monitoring Using Nonlinear Correlation Analysis of External Uterine Electromyography," IEEE Trans. Biomed. Eng., vol. 60, no. 4, pp. 1160-1166, Apr. 2013.

[4] C. Marque and J. Duchene, "Human abdominal EHG processing for uterine contraction monitoring.," Biotechnol. Read. Mass, vol. 11, pp. 187-226, 1989.

[5] M. Hassan, J. Terrien, B. Karlsson, and C. Marque, "Interactions between Uterine EMG at Different Sites Investigated Using Wavelet Analysis: Comparison of Pregnancy and Labor Contractions," EURASIP J. Adv. Signal Process., vol. 2010, no. 1, p. 918012, Jun. 2010.

[6] M. Lucovnik et al., "Noninvasive uterine electromyography for prediction of preterm delivery," Am. J. Obstet. Gynecol., vol. 204, no. 3, p. 228.e1-10, Mar. 2011.

[7] C. Rabotti, M. Mischi, S. G. Oei, and J. W. M. Bergmans, "Noninvasive Estimation of the Electrohysterographic ActionPotential Conduction Velocity," IEEE Trans. Biomed. Eng., vol. 57, no. 9, pp. 2178-2187, Sep. 2010.

[8] M. Hassan et al., "Identification of Interictal Epileptic Networks from Dense-EEG," Brain Topogr., Aug. 2016.

[9] O. Hauk, D. G. Wakeman, and R. Henson, "Comparison of noise-normalized minimum norm estimates for MEG analysis using multiple resolution metrics," NeuroImage, vol. 54, no. 3, pp. 19661974, Feb. 2011.

[10] C. Marque, A. Diab, J. Laforêt, M. Hassan, and B. Karlsson, "Dynamic Behavior of Uterine Contractions: An Approach Based on Source Localization and Multiscale Modeling," in Knowledge and Systems Engineering, V.-H. Nguyen, A.-C. Le, and V.-N. Huynh, Eds. Springer International Publishing, 2015, pp. $527-$ 540.

[11] A. Gramfort, T. Papadopoulo, E. Olivi, and M. Clerc, "OpenMEEG: opensource software for quasistatic bioelectromagnetics," Biomed. Eng. Online, vol. 9, p. 45, 2010.

[12] B. Karlsson, J. Terrien, V. Gudmundsson, T. Steingrimsdottir, and C. Marque, "Abdominal EHG on a 4 by 4 grid: mapping and presenting the propagation of uterine contractions," in 11th Mediterranean Conference on Medical and Biomedical Engineering and Computing 2007, T. Jarm, P. Kramar, and A. Zupanic, Eds. Springer Berlin Heidelberg, 2007, pp. 139-143.

[13] M. Hassan, S. Boudaoud, J. Terrien, B. Karlsson, and C. Marque, "Combination of Canonical Correlation Analysis and Empirical Mode Decomposition Applied to Denoising the Labor Electrohysterogram," IEEE Trans. Biomed. Eng., vol. 58, no. 9, pp. 2441-2447, Sep. 2011.

[14] M. Yochum, J. Laforêt, and C. Marque, "An electromechanical multiscale model of uterine pregnancy contraction," Comput. Biol. Med., vol. 77, pp. 182-194, Oct. 2016.

[15] A. Mheich et al., "A novel algorithm for measuring graph similarity: Application to brain networks," in 2015 7th International IEEE/EMBS Conference on Neural Engineering (NER), 2015, pp. $1068-1071$.

[16] M. Rubinov and O. Sporns, "Complex network measures of brain connectivity: Uses and interpretations," NeuroImage, vol. 52, no. 3, pp. 1059-1069, Sep. 2010.

[17] L. C. Freeman, "Centrality in social networks conceptual clarification," Soc. Netw., vol. 1, no. 3, pp. 215-239, 1978. 
